\title{
AN ESTIMATE OF THERMO-PHYSICAL CHANGES IN HYBRID BASALT/GLASS FIBRES REINFORCED POLYMER COMPOSITES
}

\author{
MOTOC Luca Dana ${ }^{1, a^{*}}$, BEDO Tibor ${ }^{2, b}$
}

\author{
${ }^{1}$ Transilvania University of Brasov, Material Science Department, 29 Eroilor Av., Romania \\ ${ }^{2}$ Transilvania University of Brasov, Material Science Department, 29 Eroilor Av., Romania \\ adanaluca@unitbv.ro, bedo.tibor@unitbv.ro
}

Keywords: hybrid, synthetic, polymer, fibre, basalt, thermal dilatation.

\begin{abstract}
The paper aims to provide an insight into the thermo-physical changes of in-situ tailored hybrid polymer based composite materials based on two different synthetic reinforcements with the aim of sizing both architecture stacking sequences and reinforcement type's influence on their effective linear coefficient of thermal dilatation (CTE).

The samples were subjected to a step ramp temperature increase up to $250{ }^{\circ} \mathrm{C}$, followed by their CTE variation monitoring and assessment. Data processing and comparison further contribute to expand knowledge and appropriate personal database aiming material design, manufacturing error minimization and cost reduction.
\end{abstract}

\section{Introduction}

Thermo-physical changes are being tracked for composite polymer based architectures as one of the main material properties accounted in various structural applications. Either thermal expansion or conductivity, these provide an insight into the constitutive compatibility and synergetic behaviour to be able to withstand different external environments.

Literature reports several contributions on composite architectures developed around either distributed or stacked and combined various material layers with the aim of tailoring their effective properties. The aforementioned were carried out to address: improved material properties (e.g. mechanical, dynamical, thermal and electrical, etc.), different engineering applications (e.g. transport, energy, sporting goods, etc.) or procedures applied for error minimization while designed for/from manufacturing stages.

Thus, Motoc Luca and co-authors reported the thermo-physical changes recorded for few hybrid polymers based composite architectures made as different particle (e.g. ceramic, metal) reinforced with random E-glass fibres within an unsaturated polyester resin [1], [3]. They monitored and debated on the structural changes and the material property under discussion after subjecting the hybrid composite specimens to different extreme environmental conditions, including low temperatures and temperature cycling according to the desert environment. They concluded that individual constitutive loading, external conditions and experimental parameters can be ranked as the main influencing factors upon the thermo-physical changes of the hybrid composite specimens.

Dhakal [4] and Muralidhar [5] and co-authors captured attention with their hybrid polymer composite architectures designed for improved material's properties and made from natural (e.g. flax) and synthetic (e.g. carbon, glass) reinforcements. According to their studies, layer stacking sequence and constitutive material type contributes to the effective monitored material property and further leads to improved values compared with a reference specimen.

The herein contribution presents a comparative study centred on the design, development and material characterization of in-situ manufactured hybrid polymer composite architectures reinforced with two different synthetic fibres, basalt and glass. The latter is characterized further as having two different distributions, random and woven. The results can be exploited in manufacturing cost reduction while tailoring polymer based composite architectures for various structural applications. 


\section{Materials and experimental procedure}

The composite specimens have been manufactured as a 5-ply laminate using synthetic materials like glass fibre chopped strand mats (n. GF_mat; MultiStrat ${ }^{\mathrm{TM}}$ Mat ES 33-0-25, Johns Manville, USA) and glass fibre woven fabrics (n. GF_woven; same as before), and mineral derived type like basalt fibres (n. BF; DBF Deutsche Basalt Faser GmbH, D). The reinforcements were bounded together by an epoxy resin (Epikote MGS 253, Momentive, USA) cured at room temperature by a hardener (Epikure MGS LH 233-238) for $24 \mathrm{~h}$ before cut into specimens for the experimental tests. The overall fibre loading or all composite architectures was $45 \%$.

Thermo-physical changes of specimens were performed on a differential dilatometer DIL 420 PC/1 from Netzsch GmbH (D), in accordance with ASTM E228-11 standard. The temperature mode was set up as a dynamic heating ramp trend from $25^{\circ} \mathrm{C}$ up to $250^{\circ} \mathrm{C}$. The heating rate imposed was $2.5^{\circ} \mathrm{K} / \mathrm{min}$. Two successive thermal scans were applied to remove volatiles and stress fields induce during the manufacturing step.

\section{Property prediction and modelling}

In this section a theoretical expression derived from micromechanics principles will be employed to provide an estimate on the thermal property under the focus. Contributors like Schapery, Hopkins-Chamis, Chamis and Chamberlain developed the same expression in order to estimate the linear coefficients of thermal expansion along the longitudinal direction of composite's reinforcements. Strength of materials or energy principles was set up as the framework for their assumptions and further developments. According to these models, the linear coefficients of expansion in longitudinal direction can be written as follows [6]:

$$
\alpha_{c}=\frac{\alpha_{f} E_{f} V_{f}+\alpha_{m} E_{m} V_{m}}{E_{f} V_{f}+E_{m} V_{m}}
$$

where $\alpha_{C}, \alpha_{f}$ and $\alpha_{m}$ are the linear coefficients of expansion for the effective composite, fibres and matrix material, in $\mathrm{K}^{-1}, \mathrm{E}_{\mathrm{f}}$ and $\mathrm{E}_{\mathrm{m}}$ the elastic modulus of fibres and matrix, respectively, in $\mathrm{GPa}, \mathrm{V}_{\mathrm{f}}$ and $\mathrm{V}_{\mathrm{m}}$ being the volume fraction of the constitutive.

With respect to the developed expressions to estimate expansion in transversal directions of the reinforcements, these differ from the model to model, and not employed herein due to the reinforcements' distribution.

With respect to the thermal property under focus, the mean values of the coefficient of linear thermal expansion will be retrieved based on the following formula:

$$
\alpha=\frac{1}{\mathrm{~L} 0} \cdot \frac{\Delta \mathrm{L}}{\Delta \mathrm{T}}
$$

where $\mathrm{L}_{0}$ is the specimen length at room temperature and $\Delta \mathrm{L}$ the change of specimen length between the temperature range $\Delta \mathrm{T}\left({ }^{\circ} \mathrm{C}\right)$. Further, technical alpha values will be computed with respect to a reference temperature, set herein to being $25^{\circ} \mathrm{C}$, using the following expression:

$$
\alpha\left(T, T_{\text {ref }}\right)=\frac{\frac{d L}{L_{0}}(T)-\frac{d L}{L_{0}}\left(T_{\text {ref }}\right)}{T-T_{\text {ref }}}
$$

where $\mathrm{T}$ is the upper temperature limit $\left({ }^{\circ} \mathrm{C}\right)$ whereas $\mathrm{T}_{\text {ref }}$ the reference temperature $\left({ }^{\circ} \mathrm{C}\right)$. 


\section{Results and discussions}

The experimental runs enabled records of the thermal strain fields for all specimens under discussion as well as temperature variations of their thermal expansion coefficient (CTE). As it can be seen, in Fig. 1 and Fig. 2 are plotted the aforementioned recorded property related dependencies over the temperature range. Experimental plotted data corresponds to the second thermal runs. In Table 1 are being provided the composite architectures, whereas in Table 2 their individual material thermal properties. Specimens based on solely basalt reinforcements were set out as reference for further comparison.

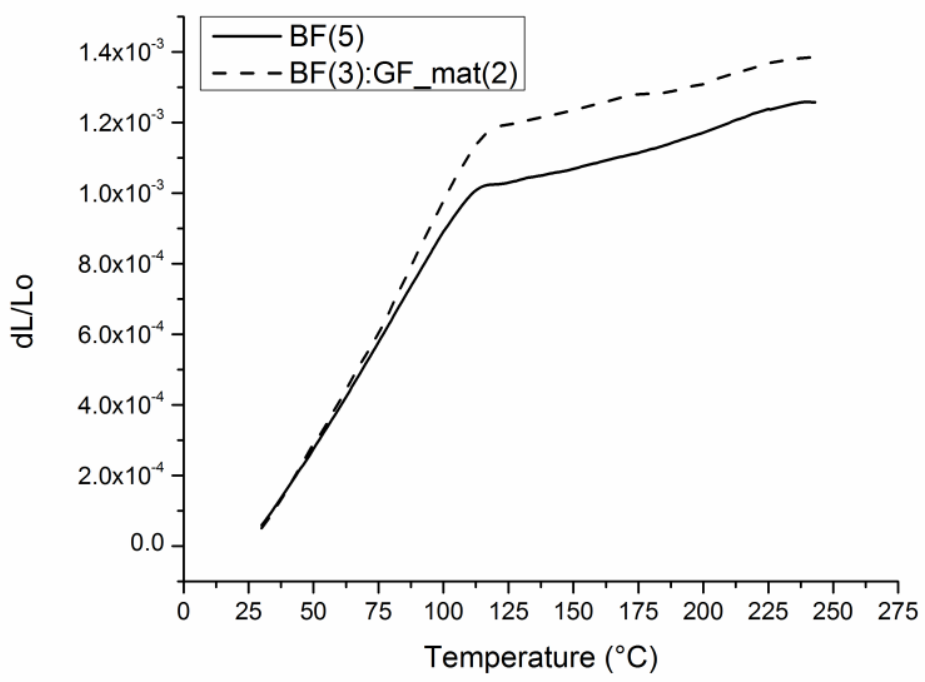

Fig. 1 Thermal strain fields evolution from the reference and hybrid composite tailored with random glass fibres

As it can be seen from Fig. 1, the recorded thermal strain fields show a linear trend up to approximate $110^{\circ} \mathrm{C}$ with an abrupt slope, region associated to the specimen expansion. For the remaining temperature range, the trend resembles again the linear tendency but with diminished slopes, the specimens expanding further with the temperature increase. The above-mentioned temperature $\left(110^{\circ} \mathrm{C}\right)$ can be regarded as the specimen's softening point.

Concerning the technical alpha, evaluated applying the formula from equation (2) the curves tendencies inverse. Thus, up to $110^{\circ} \mathrm{C}$ the variation can be regarded as almost linearly, while beyond as revealing an abrupt decrease. In the first temperature region, between $25^{\circ}$ and $110^{\circ} \mathrm{C}$, the thermo-physical changes can be assigned to constitutive and their loading within the hybrid structure as well as to the volatiles evaporation. The slope change while passing through the softening point can be ascribed to the structural relaxation within the polymer composite structures.

Table 1 - Laminates stacking sequence and hybrid ratio

\begin{tabular}{|c|c|c|c|}
\hline Matrix system & Stacking sequence & Laminate codes & Hybrid ratio (BF:GF) \\
\hline \multirow{3}{*}{ Epoxy } & $\square(a \square$ & $\mathrm{BF}(5)$ & $100: 0$ \\
\cline { 2 - 4 } & $\square$ & $\mathrm{BF}(3): \mathrm{GF}$ mat(2) & $60: 40$ \\
\cline { 2 - 4 } & $\square$ & $\mathrm{BF}(3): \mathrm{GF}$ _woven(2) & $60: 40$ \\
\hline
\end{tabular}

Table 2 - Individual material elastic and thermal properties

\begin{tabular}{|c|c|c|c|c|}
\hline \multirow{2}{*}{ Property } & \multicolumn{3}{|c|}{ Material } & Reference \\
\cline { 2 - 4 } & E-glass fibres & Basalt fibres & Epoxy & [6], [7] \\
\hline Elastic modulus $(\mathrm{GPa})$ & 75 & 110 & 2.5 & \\
\hline CTE $\left(\times 10^{-5} \mathrm{~K}^{-1}\right)$ & 5 & 5.5 & 3 & \\
\hline
\end{tabular}




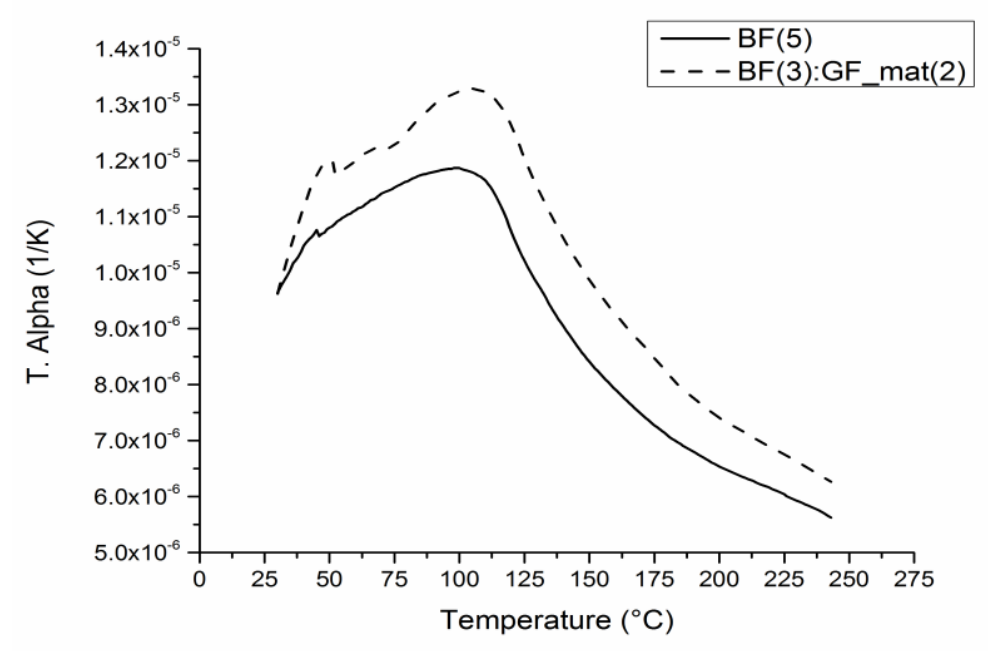

Fig. 2 Instantaneous technical alpha variation with temperature for the reference and hvbrid polvmer composite

In Fig. 3 is being plotted the relative difference errors between the experimentally recorded technical alpha values of the hybrid composite architectures with respect to the reference sample from both thermal scan records. Discrepancies between values can be regarded to the glass fibres reinforcement type, namely their orientation and density. The aforementioned is assigned to the lowmass density of the woven glass fibres used in $\mathrm{BF}(3)$ :GF_woven(2) hybrid composite architecture that is loosening enough space for expansion. Thermal expansion of basalt fibre dominates the overall expansion of the hybrid composites.

Further, discrepancies can be seen between the experimentally retrieved and theoretical predicted values as plotted in Fig. 4. Firstly, as it can be seen, there are small differences between theoretical and experimental values in case of the reference based solely on basalt reinforcements. This can be regarded to the individual materials properties used (see Table 2) that are consistent with the experimental values.

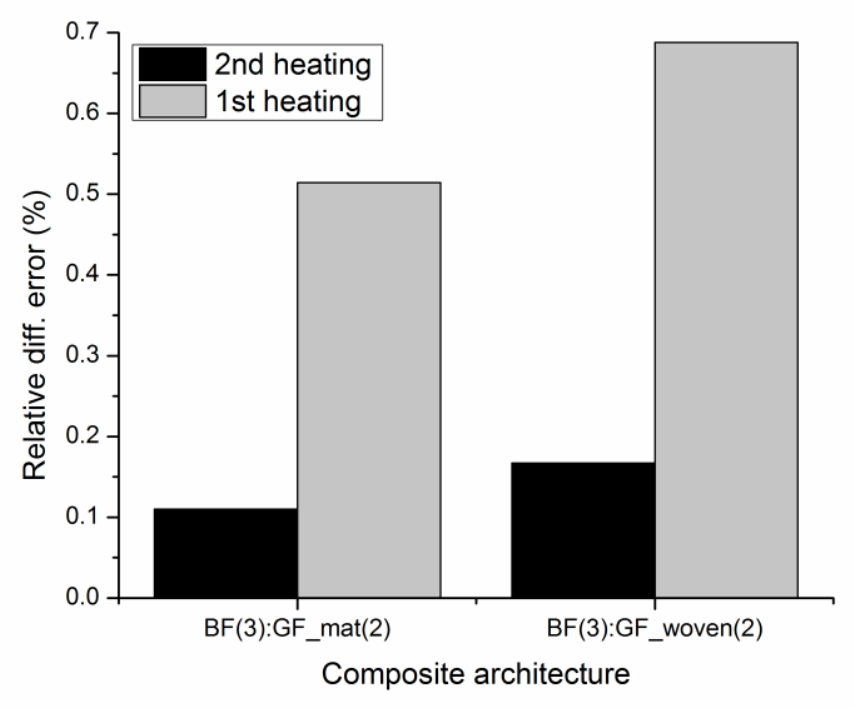

Fig. 3 Relative difference errors between the experimentally retrieved technical CTE from both thermal scans for hybrid composite architectures with respect to the reference 


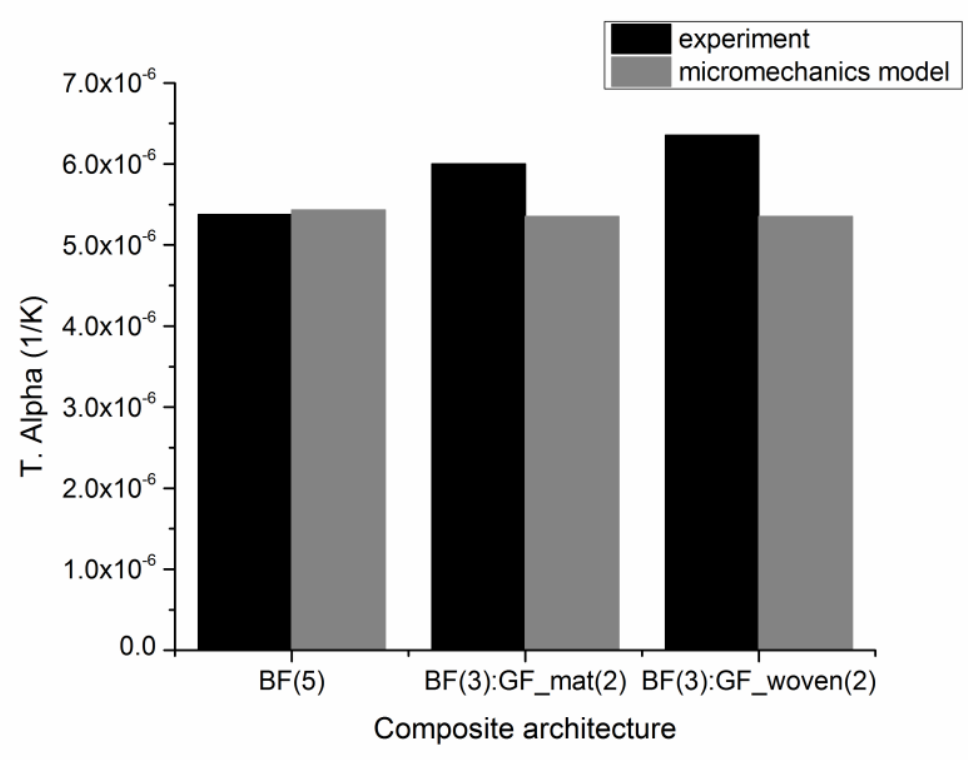

Fig. 4 Experimentally retrieved and micromechanically predicted CTE values for all composite architectures

Next, the different glass fibre orientations seem to indicate small discrepancies between the predicted and retrieved values. As it can be seen, theoretical predictions provide an identical value of the effective thermal property due to employment of the same individual properties' values for glass fibre reinforcements in both cases. It is beyond the purpose of this article to investigate the effect of fibre orientation and use/development of other theoretical models in order to encompass this.

In Fig. 5 is being represented the percent relative error values between the theoretical predicted and experimentally retrieved CTE values for all composite architectures under debate. Accordingly, to be above mentioned, the differences are relatively high (approx. 18\%) for the woven glass reinforcement multi-phase architecture and very small for $\mathrm{BF}(5)$ polymer composite (approx. 1\%). Again, the previous identified influencing factor on the effective CTE reveal its effects on this percent relative error values.

It must be underlined that the aforementioned holds for the experimentally CTE values retrieved from the second thermal runs. These values can be regarded as free of volatiles and other thermal effects or history influence.

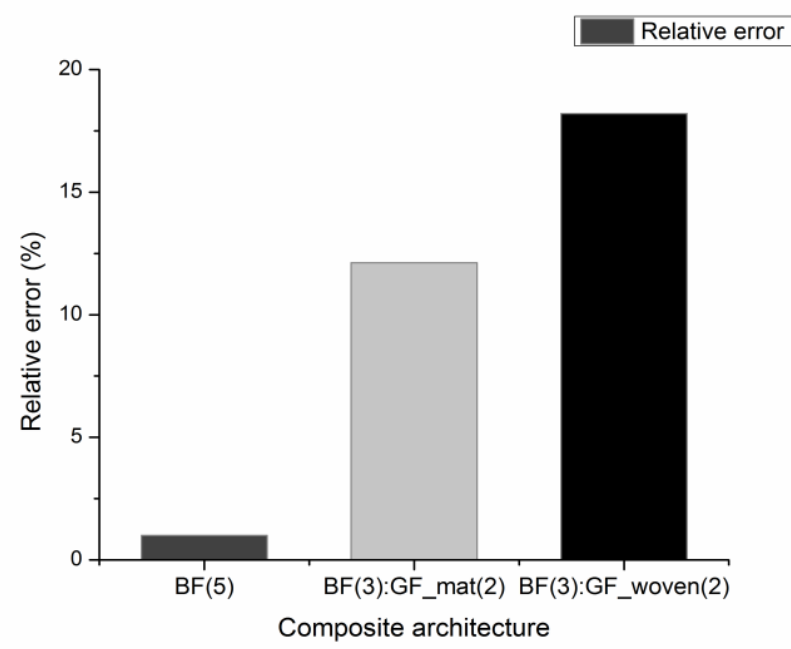

Fig. 5 Difference error between predicted and exprimentally retreived CTE values for all composites 


\section{Conclusions}

The paper attempted to approach the thermo-physical changes in few two-phase and multi-phase polymer reinforced composite architectures tailored by different stacking their constitutive. Furthermore, the study explored the possibility of using basalt fibres in combination with glass fibres and investigated the effect of stacking sequence and different orientation of the latter on one of the most important thermal properties for a material. This could provide an opportunity to using basalt/glass epoxy composite laminates for applications where heat/fire withstanding is among the primary design requirements.

To sum up, all the observations and findings, it can be acknowledged that:

- the multi-phase composite reinforced with woven glass laminates revealed the highest value of the linear coefficient of expansion;

- discrepancies in CTE values between the different glass fibre oriented composite architectures can be regarded to the fibres' mass densities that are loosening space for the other fibres to expand as temperature increases;

- for the same fibre loading, either basalt or glass, stacked identically, there are small differences among their experimentally retrieved CTE values;

- in all cases, a positive hybridization effect with respect to the thermal property under debate was encountered;

This study provides several perspectives and route of actions that can be followed in order to characterize these hybrid architectures, including mechanical, dynamical or electrical property retrieval. Supplementary, if acoustical properties are being sought, application in construction and buildings of these composites can be easily foreseen.

With respect to the micro-mechanical models, there is a stringent need for other predictive theoretical models that may allow a better approach and insight into the complex structure particular about these hybrid composite architectures.

\section{References}

[1] D. Luca Motoc, I. Curtu, et. al, Multiphase polymeric composite materials CTE variation with extreme environmental conditions, Mat. Plast., 47 (2010) 236-239.

[2] D. Luca Motoc, N. Dadirlat, Particle size and structural composition influences on the overall CTE behavior of recycled polymer composites, Metal. Int., XVI (2011) 149-152.

[3] D. Luca Motoc, J. Ivens, N. Dadirlat, Coefficient of thermal expansion evolution for cryogenic preconditioned hybrid carbon fiber/glass fiber reinforced polymeric composite materials, J. Therm. Anal. Calorim., 112 (2013) 1245-1251.

[4] H. N. Dhakal, Z. Y. Zhang, R. Guthrie, J. MacMullen, N. Bennett, Development of flax/carbon fibre hybrid composites for enhanced properties, Carb. Polym., 96 (2013) 1-8.

[5] B. A. Muralidhar, Study of flax hybrid preforms reinforced epoxy composites, Mat. \& Design (2013) 835-840.

[6] I. Curtu, D. Luca Motoc, Micromecanica materialelor compozite, Ed. Universitatii Transilvania din Brasov, 2009.

[7] B. Rice, A. C. Rice, Young's modulus and thermal expansion of filled cyanat ester and epoxy resins, IEEE Trans. On Appl. Supercond. (2009) 2371-2374. 